\title{
Efficient Calculation of Exact Mass Isotopic Distributions
}

\author{
Ross K. Snider \\ Snider Technology, Inc., Bozeman, Montana, USA, and Department of Electrical and Computer Engineering, \\ Montana State University, Bozeman, Montana, USA
}

This paper presents a new method for efficiently calculating the exact masses in an isotopic distribution using a dynamic programming approach. The resulting program, isoDalton, can generate extremely high isotopic resolutions as demonstrated by a FWHM resolution of $2 \times 10^{11}$. This resolution allows very fine mass structures in isotopic distributions to be seen, even for large molecules. Since the number of exact masses grows exponentially with molecular size, only the most probable exact masses are kept, the number of which is user specified. (J Am Soc Mass Spectrom 2007, 18, 1511-1515) (c) 2007 American Society for Mass Spectrometry

$\mathrm{F}$ or most practical applications, computing the very fine isotopic structure is unnecessary as it is beyond the resolution of today's mass spectrometers. However, for theoretical considerations, it is useful to have a method that can calculate the exact mass distributions of these fine structure clusters, which could prove useful as the resolution of mass spectrometers improves. One of the challenges in calculating distributions is maintaining a very high-resolution around isotope peaks and yet being computationally efficient.

A number of methods [1-9] have been employed to elucidate the fine isotopic structure. The majority are polynomial based methods that rely on pruning to reduce the complexity to a more manageable size. The pruning strategies typically use a threshold to eliminate permutations whose contribution falls below some preset value. This creates errors in the isotopic distribution profile since a significant number of terms are eliminated.

Rockwood et al. [8, 9] use a Fourier transform method to zoom in and achieve ultrahigh resolution around a single mass peak. As with any Fourier analysis method, care must be taken to choose an appropriate window function. The windowing will cause the underlying Dirac delta functions representing fine isotopic masses to be convolved together if they are closer together than the width of the analysis window.

A new method based on dynamic programming [10] is presented that can efficiently calculate isotopic distributions. In principle, the resolution is infinite, since there is no restriction on how close in mass the states can be to each other. In practice, the resolution seen depends on machine precision and the probability of the mass states. Low probability states that are very

Address reprint requests to Dr. R. K. Snider, Snider Technology, Inc., Bozeman, MT 59715, USA. E-mail: ross@snidertech.com close to other more probable states may either be eliminated or merged, depending on the state reduction strategy employed.

This method can operate like a polynomial pruning method if low probability states are eliminated. If neighboring states are merged instead, it can operate more like the Fourier transform method. In the Fourier transform method, all peaks under the window contribute, whereas the merging in the dynamic programming case is local.

The implementation of the algorithm is a program called isoDalton (see Supplementary Material section, which can be found in the online version of this article.) that has been written in MATLAB [12] and is freely available under the GNU Lesser General Public License [13], which allows use in both proprietary and free programs. The program includes all the isotopes of all the elements with the standard isotopic compositions [14]. Custom isotopic compositions can be easily added.

\section{Algorithm Description}

Calculating ion distributions for large molecules require expanding the polynomial of the form

$$
\begin{aligned}
& \left(E_{1}^{1}+E_{2}^{1}+\cdots+E_{I_{1}}^{1}\right)^{N_{1}}\left(E_{1}^{2}+E_{2}^{2}+\cdots+E_{I_{2}}^{2}\right)^{N_{2}} \\
& \quad \times\left(E_{1}^{3}+E_{2}^{3}+\cdots+E_{I_{3}}^{3}\right)^{N_{3}} \cdots
\end{aligned}
$$

where $E_{j}^{i}$ represents the $\mathrm{j}^{\text {th }}$ isotope of the $\mathrm{i}^{\text {th }}$ element in the molecule. The $N_{i}$ superscript outside the parenthesis represents the number of atoms of the $i^{\text {th }}$ element [4]. This will generate a combinatorial explosion in the number of terms for large molecules. The number of coefficients for the multinomial $\left(E_{1}^{i}+E_{2}^{i}+\cdots+E_{I_{i}}^{i}\right)^{N_{i}}$ representing the $\mathrm{i}^{\text {th }}$ element with $N_{i}$ atoms and $\mathrm{I}_{\mathrm{i}}$ isotopes is given by [15]: 


$$
C_{I_{i}}^{N_{i}}=\frac{\left(N_{i}+I_{i}-1\right) !}{N_{i} !\left(I_{i}-1\right) !}
$$

and the coefficients of the multinomial are given by:

$$
\begin{aligned}
\left(E_{1}^{i}\right. & \left.+E_{2}^{i}+\cdots+E_{I_{i}}^{i}\right)^{N_{i}} \\
& =\sum_{M_{1}+M_{2}+\cdots M_{I_{i}}=N_{i}} \frac{N_{i} !}{M_{1} ! M_{2} ! \cdots M_{I_{i}} !} E_{1}^{i M_{1}} E_{1}^{i M_{2}} \cdots E_{1}^{i M_{I_{i}}}
\end{aligned}
$$

The total number of terms $\mathrm{T}$ in the expanded polynomial of eq. 1 is the number of terms in the product of the elemental multinomial coefficients and is given by:

$$
T=C_{I_{1}}^{N_{1}} C_{I_{2}}^{N_{2}} C_{I_{3}}^{N_{3}} \ldots
$$

which gives the number of possible masses in the isotopic fine structure.

For bovine insulin $\mathrm{C}_{254} \mathrm{H}_{377} \mathrm{~N}_{65} \mathrm{O}_{75} \mathrm{~S}_{6}$ the number of possible terms is $1.56 \times 10^{12}$, which clearly precludes any brute force attack. In practice, one only needs a fraction of the terms since most of the terms are extremely unlikely. The least probable term is ${ }^{13} \mathrm{C}_{254}{ }^{2} \mathrm{H}_{377}{ }^{15} \mathrm{~N}_{65}{ }^{17} \mathrm{O}_{75}{ }^{35} \mathrm{~S}_{6}$ that has a probability of $0.2610 \times 10^{-2422}$ of occurring. In fact, the top 1000 terms represents $99.96 \%$ of the cumulative probability distribution.

An efficient method based on dynamic programming can be used to calculate the overall distribution of possible molecular weights given the isotopic distribution for each element. To apply dynamic programming, we first frame this calculation in the context of a Markov process $\left\{X_{t}\right\}_{t \in T}$ operating on a discrete state space $S$. The state transition probabilities are given by:

$$
p_{i j}=P\left(X_{n+1}=j \mid X_{n}=i\right), \quad n \geq 0, i, j \in S
$$

This gives the probability of arriving at state $S_{j}$ at step $n+1$, given that it was in state $S_{i}$ at step $n$. The state transition probabilities are required to have the following properties:

$$
\begin{aligned}
& p_{i j} \geq 0 \\
& \sum_{j=1}^{I} p_{i j}=1, \quad \forall i, j=1, \ldots, J .
\end{aligned}
$$

The initial state probabilities are given by:

$$
\pi_{i}(0)=P\left(X_{0}=i\right), \quad i \in S
$$

The efficient way to calculate the probability of being in state $S_{j}$ at step $n+1$ is to use a forward trellis algorithm [16]. An illustration of this computation can be seen in Figure 1. by

The state probabilities for step $n+1$ are calculated

$$
\alpha_{S_{j}}(n+1)=\sum_{i=1}^{N(n)} \alpha_{S_{j}}(n) p_{i j}
$$

where $1 \leq j \leq N(n+1), 1 \leq n \leq \mathrm{T}-1 . \mathrm{N}(n)$ implies that the number of states is a function of step $n$.

The trellis algorithm gains its efficiency by collapsing the possible paths that can lead to a particular state. Only the state probabilities at step $\mathrm{n}$ along with the transition probabilities are used to calculate the state probabilities for the next step. This is known as a first-order Markov model or chain [17].

In the context of calculating the isotope distribution, the states are the set of unique molecular masses that can exist at each step. At each step, all isotopes of

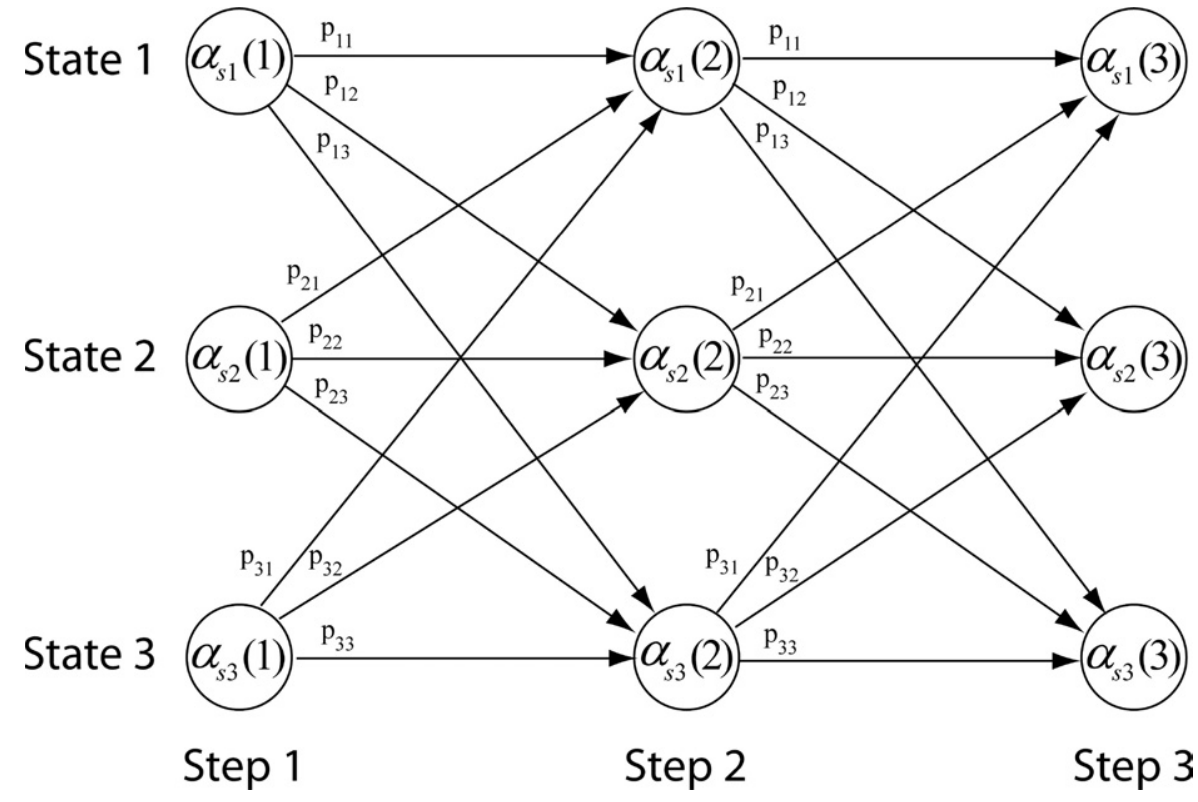

Figure 1. Trellis for efficiently computing state probabilities. 
one atom of a particular element are added, i.e., $\left(E_{1}^{i}+E_{2}^{i}+\cdots+E_{I_{1}}^{i}\right)^{1}$. This means that the state transition probabilities are non-stationary since they depend on the isotope distribution of the particular element being added. The Markov chain can be thought of as the sequence of adding elements with all associated isotopes at each step. The length of the chain is the number of elements in the molecule.

The number of states at each step is also nonstationary since particular combinations of isotopes lead to unique masses. The states at step $n+1$ is the set of unique masses computed by adding the mass of any state at step $\mathrm{n}$ with any isotope of the element being added. The probabilities of these states are given in eq 8. These states are then either pruned or combined to reduce computational complexity and this process is called state reduction.

\section{State Reduction}

\section{Most Probable Exact Masses}

Keeping the distribution of all exact masses becomes impractical for all but the smallest molecules. If one is interested in the exact masses of the most probable isotope mass combinations, as is typically the case, then the states with lowest probabilities are eliminated. This is done by computing all states for step $n+1$, sorting these states based on probabilities, and then keeping only the top $\mathrm{N}_{\max }$ most probable states where $\mathrm{N}_{\max }$ is user specified. Once all the elements have been added at the last step, isoDalton returns the exact masses of the $\mathrm{N}_{\max }$ most probable isotopic mass combinations. The "true" probabilities of these exact masses are only approximations since eliminating states prunes potential path combinations that affect probability values. Increasing $\mathrm{N}_{\max }$ will reduced this error.

\section{Exact Probability Distribution}

If one is interested in seeing the overall probability distribution of near integer separated values, then close mass values can be combined as follows. Let $M_{\text {old1 }}$ and $M_{\text {old2 }}$ be the masses of states $S_{i}$ and $S_{j}$ that are the closest together in terms of mass values and let $\mathrm{P}_{\text {old1 }}$ and $\mathrm{P}_{\text {old2 }}$ be their respective probabilities. Then a new state is created that has mass and probability of:

$$
\begin{aligned}
& M_{\text {new }}=\left(M_{\text {old } 1} P_{\text {old } 1}+M_{\text {old } 2} P_{\text {old } 2}\right) /\left(P_{\text {old } 1}+P_{\text {old } 2}\right) \\
& P_{\text {new }}=P_{\text {old } 1}+P_{\text {old } 2}
\end{aligned}
$$

For a particular step $n$, the states are combined in this fashion until there are $\mathrm{N}_{\max }$ states. Combining states in this manner results in a probability distribution of $\mathrm{N}_{\max }$ masses that are the center of masses of the isotopic fine structure exact masses. These are exact probabilities for these "center of mass" weights since they sum to 1 as expected of a probability distribution. However, the masses are not exact.

\section{Results and Discussion}

As an example, we find the complete molecular weight distribution of the amino acid glycine, $\mathrm{C}_{2} \mathrm{H}_{5} \mathrm{~N}_{1} \mathrm{O}_{2}$ (including the amino and carboxyl end groups). We view the Markov process as the sequence of adding the elements $\mathrm{H}, \mathrm{H}, \mathrm{H}, \mathrm{H}, \mathrm{H}, \mathrm{C}, \mathrm{C}, \mathrm{N}, \mathrm{O}$, and $\mathrm{O}$, where the order of elements is taken by starting with the element with the fewest number of isotopes. Starting with elements with fewest isotopes minimizes the growth in the number of states. The isotopes used in this example are the NIST values [14].

The initial state probability $\pi(0)$ is simply the isotopic composition of hydrogen $\{0.999885,0.000115\}$ at states (masses) \{1.0078250321, 2.0141017780\}. To compute the new state probabilities, we add another hydrogen and compute the resulting states and probabilities of being in these states. The new states at step $n+1$ are all permutations of adding the molecular weights of the states at step $\mathrm{n}$ with all the isotopes of element $\mathrm{E}^{\mathrm{i}}$.

The atomic weights for all states at each step can be seen in Figure 2. This was created by adding the glycine elements $\mathrm{H}, \mathrm{H}, \mathrm{H}, \mathrm{H}, \mathrm{H}, \mathrm{C}, \mathrm{C}, \mathrm{N}, \mathrm{O}, \mathrm{O}$.

A more abstract view of the trellis can be accomplished by finding at each step the state with the minimum atomic weight, and then subtracting this value from all states for this step. Figure 3 shows this abstracted view where the distances between states can be more clearly seen. The bottom row in the figure shows which element was added at each step. Above the trellis, the number of states is given that exist for each step. We start off with two states since hydrogen has two isotopes and we end up with 216 states or distinct atomic weights after adding all 10 elements. Many of the states are clustered together near unitary atomic weight increments. In practice, only the states at

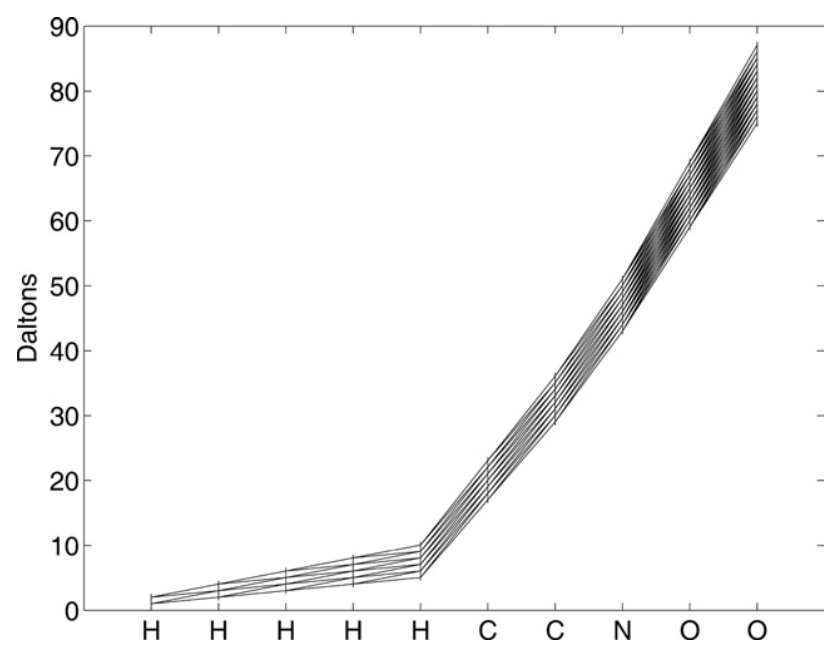

Figure 2. Trellis showing all mass states for glycine $\mathrm{C}_{2} \mathrm{H}_{5} \mathrm{~N}_{1} \mathrm{O}_{2}$. 


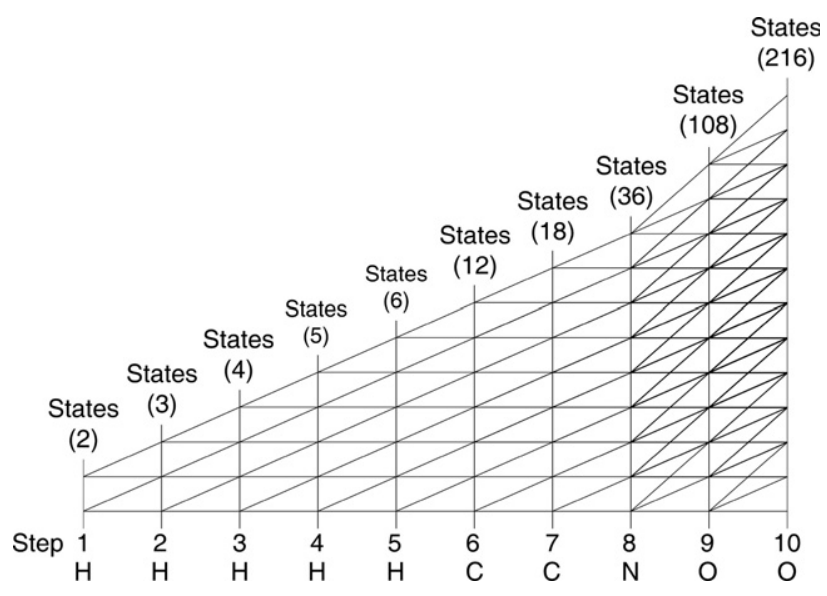

Figure 3. Abstract view of trellis formation for glycine $\mathrm{C}_{2} \mathrm{H}_{5} \mathrm{~N}_{1} \mathrm{O}_{2}$.

step $n$ are kept since the state history is not needed and would only consume memory.

The complete molecular weight distribution of glycine can be seen in Figure 4. The distribution is plotted with two different scales. Positive values are the probabilities of isotopic masses where the scale is on the left upper side. Since most of the probabilities are very small, a $\log$ scale is plotted by taking the $\log 10$ of the probabilities. This results in negative numbers that are seen in the lower half of the plot with the scale on the right lower side. There are 216 unique mass values in this plot that are clustered near 13 integer values. There is a single dominant peak at mass 75.03 with probability 0.97 . There are four mass values clustered at 76.03 and are shown in the inset plot. There is a very small single peak at mass 87.08 with probability $3.56 \times 10^{-32}$ and can be seen clearly on the $\log 10$ scale with value -31.4484 . The closest states occur at 81.05 with a separation of $7.21 \times 10^{-5}$, which means a FWHM resolution of $1.12 \times 10^{6}$ is needed to resolve these peaks.

For large molecules, keeping all unique states will cause the number of states to grow too large for memory and speed considerations. To keep the states small and still have a useful distribution, one can combine the states by adding clustered weights together. This is done by adding all the probabilities together in a cluster. In the glycine example, this would reduce the number of final states from 216 to 13 . As a result, one can trade off distribution precision for a reduction in computational complexity.

To show the program's utility with large molecules, bovine insulin $\mathrm{C}_{254} \mathrm{H}_{377} \mathrm{~N}_{65} \mathrm{O}_{75} \mathrm{~S}_{6}$ was chosen for comparison purposes with previous publications. Figure 5 shows the fine isotopic structure around the $5736.6 \mathrm{Da}$

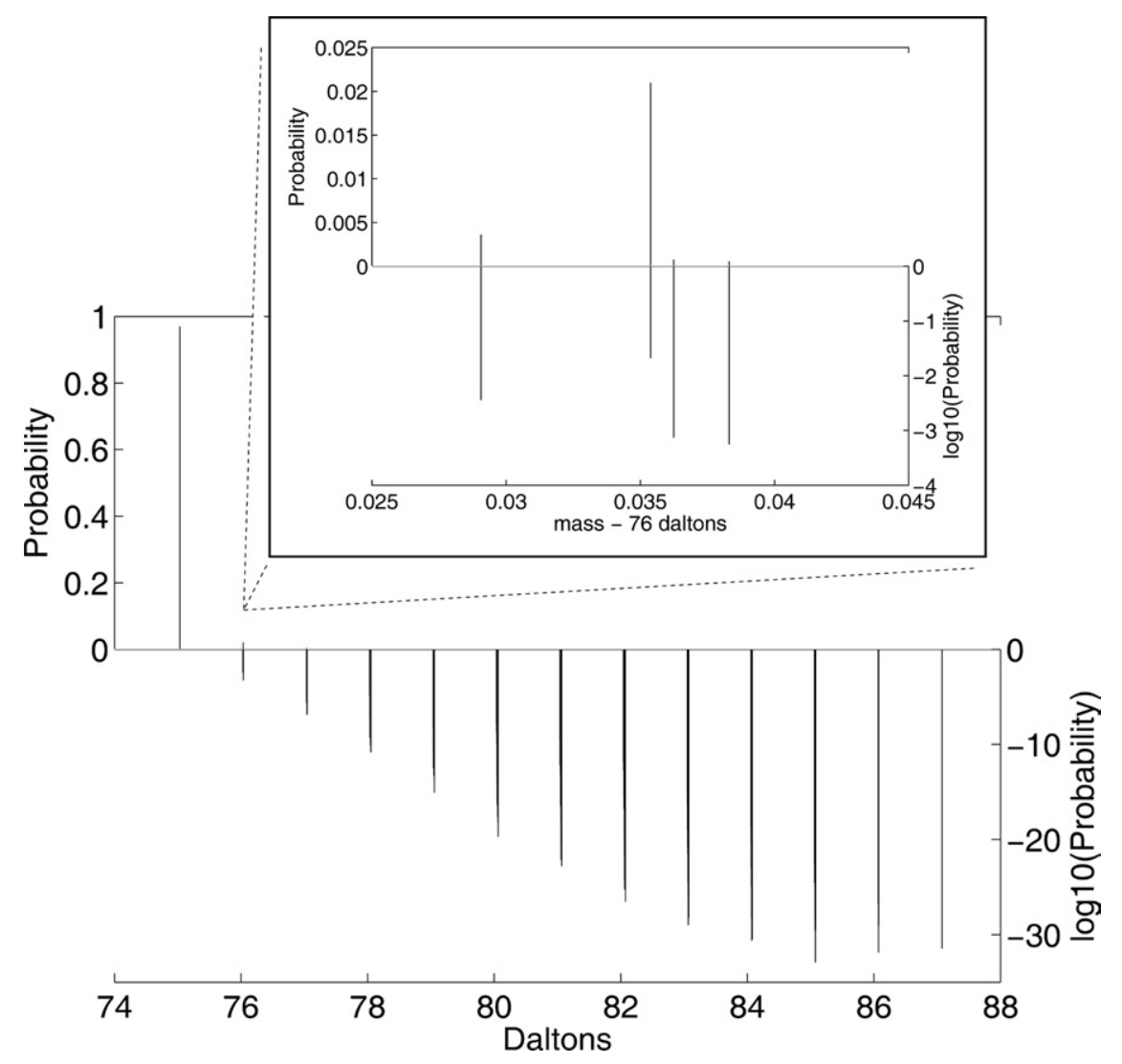

Figure 4. Complete isotopic distribution of glycine $\mathrm{C}_{2} \mathrm{H}_{5} \mathrm{~N}_{1} \mathrm{O}_{2}$. There are 216 unique masses of which the probabilities are shown on two scales. These two scales are the typical probability scale (upper left) and the $\log 10$ probability scale (lower right) that show the improbable peaks (large negative log values). The inset plot shows 4 peaks that comprise the 76.03 Da isotopic cluster. 
peak of protonated bovine insulin with a FWHM resolution of $2 \times 10^{11}$, which is a resolution of several orders of magnitude finer than previously shown. This distribution was calculated by keeping the top 100,000 most probable states, 416 of which are near the 5736.6 peak. The resolution is similar for the other isotopic clusters that range in mass from 5730.6 to 5759.7 .

\section{Run Times}

There is a tradeoff between execution speed and the FWHM resolution. Table 1 shows the tradeoff between the number of states and the run time for exact mass calculations. The time per state actually gets better with additional states due to a relatively fixed overhead of processing the state vectors in the program.

\section{Conclusions}

The paper presents a new method based on dynamic programming that can calculate the distribution of exact masses in an isotopic distribution. The resulting MATLAB program isoDalton can be used to calculate the isotopic

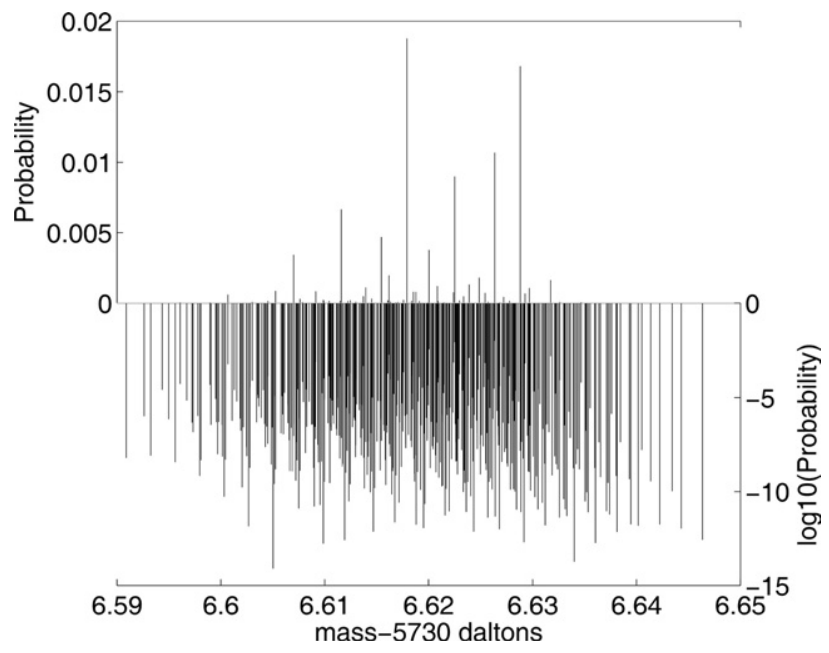

Figure 5. Isotopic fine structure of the 5736.6 peak of protonated bovine insulin $\mathrm{C}_{254} \mathrm{H}_{377} \mathrm{~N}_{65} \mathrm{O}_{75} \mathrm{~S}_{6}$ shown with a FWHM resolution of $2 \times 10^{11}$. The distribution is plotted against two scales. The scale on the top left plots the probability of mass terms. However, most of the mass terms have very low probabilities and do not show up in the probability plot since they are essentially zero. To show these terms, a $\log 10$ scale is shown on the bottom right. Each mass term is shown in each scale, at the same mass location. There are 416 distinct mass terms in this cluster from an overall distribution of the 100,000 most probable mass states.
Table 1. Run times of exact mass calculations

\begin{tabular}{lcc}
\hline States & FWHM resolution [11] & Run time $^{a}$ \\
\hline \hline 10 & $1.96 \times 10^{6}$ & $0.7 \mathrm{sec}$ \\
100 & $1.42 \times 10^{7}$ & $3.9 \mathrm{sec}$ \\
1,000 & $4.68 \times 10^{8}$ & $25 \mathrm{sec}$ \\
10,000 & $1.96 \times 10^{10}$ & $3.5 \mathrm{~min}$ \\
100,000 & $2.01 \times 10^{11}$ & $29.6 \mathrm{~min}$ \\
\hline
\end{tabular}

aIntel Core 266002.4 GHz, 4 GB RAM, Matlab 7.3.0.267 (R2006b).

fine structure of large molecules with extremely highresolution. This is done by keeping only the most probable states representing exact masses. The program can also calculate isotopic distributions with a true probability profile at the expense of knowing exact masses by merging very close mass states.

\section{Acknowledgments}

This work was supported by the National Institutes of Health grant 1R43HG003743-01.

\section{References}

1. Werlen, R. C. Effect of Resolution on the Shape of Mass Spectra of Proteins: Some Theoretical Considerations. Rapid Commun. Mass Spectrom. 1994, 8, 976-980.

2. Yergey, J. A. A General Approach to Calculating Isotopic Distributions for Mass Spectrometry. Int. J. Mass Spectrom. Ion Phys. 1983, 52 337-349.

3. Kubinyi, H. Calculation of Isotope Distributions in Mass Spectrometry. A Trivial Solution for a Nontrivial Problem. Anal. Chim. Acta. 1991, 247, 107-119.

4. Brownawell, M. L.; San Filippo, J. A Program for the Synthesis of Mass Spectral Isotopic Abundances. J. Chem. Edu. 1982, 59(8), 663-665.

5. Roussis, S. G.; Prouix, R. Reduction of Chemical Formulas from the Isotopic Peak Distributions of High-Resolution Mass Spectra. Anal Chem. 2003, 75, 1470-1482.

6. Rockwood, A. L.; Van Orman, J. R.; Dearden D. V. Isotopic Composition and Accurate Masses of Single Isotopic Peaks. J. Am. Soc. Mass Spectrom. 2004, 15, 2004.

7. Rockwood, A. L.; Haimi, P. Efficient Calculation of Accurate Masses of Isotopic Peaks. J. Am. Soc. Mass Spectrom. 2006, 17, 415-419.

8. Rockwood, A. L.; Van Orden, S. L.; Smith R. D. Rapid Calculation of Isotope Distributions. Anal. Chem. 1995, 67, 2699-2704.

9. Rockwood, A. L.; Van Orden, S. L.; Smith R. D. Ultrahigh Resolution Isotope Distribution Calculations. Rapid Commun. Mass Spectrom. 1996, 10, 54-59.

10. Bellman, R. On the Theory of Dynamic Programming, Proceedings of the National Academy of Sciences 1952, 38(8), 716-719.

11. Siuzdak, G. The Expanding Role of Mass Spectrometry in Biotechnology MCC Press: San Diego, 2003; p. 44.

12. MATLAB, a technical computing environment and language from The Mathworks, Inc., www.mathworks.com.

13. Free Software Foundation, Inc. Boston, MA; http://www.gnu.org/ licenses.

14. NIST Isotope Data: http://physics.nist.gov/PhysRefData/Compositions/ index.html.

15. Mott, J. L.; Kandel, A.; Baker, T. P. Discrete Mathematics for Computer Scientists and Mathematicians, 2nd ed.; Prentice Hall: Englewood Cliffs, NJ, 1986; Chap II.

16. Ma, X.; Kavcic, A. Path Partitions and Forward-Only Trellis Algorithms. IEEE Trans. Information Theory 2003, 49(1), 38-52.

17. Rabiner, L. R. A Tutorial on Hidden Markov Models and Selected Applications in Speech Recognition. Proceedings of the IEEE 1989, 77(2), 257-286. 